\title{
Zirconia-titania-doped tantala optical coatings for low mechanical loss Bragg mirrors
}

\author{
Émile Lalande ${ }^{1}$, Alexandre W. Lussier ${ }^{1}$, Carl Lévesque ${ }^{1}$, Marianne Ward $^{1}$, Bill \\ Baloukas $^{2}$, Ludvik Martinu ${ }^{2}$, Gabriele Vajente ${ }^{3}$, Garilynn Billingsley ${ }^{3}$, Alena \\ Ananyeva $^{3}$, Riccardo Bassiri ${ }^{4}$, Martin M. Fejer ${ }^{4}$, and François Schiettekatte ${ }^{1, *}$ \\ ${ }^{1}$ Université de Montréal, Montréal, Québec, Canada \\ ${ }^{2}$ École Polytechnique de Montréal, Montréal, Québec, Canada \\ ${ }^{3}$ LIGO Laboratory, California Institute of Technology, Pasadena (CA) USA \\ ${ }^{4}$ Stanford University, Stanford (CA) USA \\ ${ }^{*}$ Corresponding author, email: francois.schiettekatte@umontreal.ca
}

May 24, 2021

\begin{abstract}
The noise caused by internal mechanical dissipation in the high refractive index amorphous thin films in dielectric mirrors is an important limitation for gravitational wave detection. The objective of this study is to decrease this noise spectral density, which is linearly dependent on such dissipation and characterized by the loss angle of the Young's modulus, by adding zirconia to titania-doped tantala, from which the current mirrors for gravitational wave detection are made. The purpose of adding zirconia is to raise the crystallization temperature, which allows the material to be more relaxed by raising the practical annealing temperature. The Ta, Ti and Zr oxides are deposited by reactive magnetron sputtering in an $\mathrm{Ar}: \mathrm{O}_{2}$ atmosphere using radio-frequency and high power impulse plasma excitation. We show that thanks to zirconia, the crystallization temperature rises by more than $150^{\circ} \mathrm{C}$, which allows one to obtain a loss angle of $2.5 \times 10^{-4}$, that is, a decrease by a factor of 1.5 compared to the current mirror high-index layers. However, due to a difference in the coefficient of thermal expansion between the thin film and the silica substrate, cracks appear at high annealing temperature. In response, a silica capping layer is applied to increase the temperature of crack formation by $100^{\circ} \mathrm{C}$.
\end{abstract}




\section{Introduction}

Gravitational wave detection opens a new window on extreme phenomena such as merging of black holes [1] and neutron stars [2]. Improvement of the detector's sensitivity would allow not only more frequent and detailed detection, but also observation of new types of stellar events.

The dominant source of noise in the most sensitive frequency range $(50-300 \mathrm{~Hz})$ of the detection experiments is a combination of the quantum noise [3] and the thermal noise; the latter finds its origin in the internal mechanical dissipation in the interferometer's mirrors through the fluctuationdissipation theorem [4]. The dissipation is characterized by a loss angle, which is proportional to the fraction of elastic energy dissipated at each cycle of oscillation. The current mirrors of the Advanced Laser Interferometer Gravitational Wave Observatory (a-LIGO) are made of Bragg reflectors with alternating layers of $\mathrm{SiO}_{2}$ (silica), which has a low refractive index, and amorphous $\mathrm{Ta}_{2} \mathrm{O}_{5}$ (tantala) doped with $\mathrm{TiO}_{2}$ (titania), which has a high refractive index. These state-of-the-art materials for interferometric filters were the subject of intense research [5] and their optical properties have been shown to be linked, for example, to their mechanical properties $[6,7]$. In addition, titania was shown to reduce the loss angle when added to tantala [8]. Still, titania-doped tantala is the material that contributes most to the thermal noise, with a loss angle of approximately $3.7 \times 10^{-4}$ compared to silica, whose loss angle is in the order of $5 \times 10^{-5}[9]$.

These values are above the design specifications of the planned upgrade to A+ LIGO for which the target is to reduce the thermal noise by a factor of 2 (hence the loss angle by a factor of 4 ) for the observation runs starting in the next few years. In order to reach this objective, the following paths are being examined: using different layer compositions, deposition tools and methods (including nanolayers), post-treatment strategies, or using mono-crystalline layers instead of amorphous ones [10, 11]. Recently, Fazio et al. [12] were able to reach a loss angle of $2.8 \times 10^{-4}$ in $27 \%$ Ti-doped tantala deposited by ion beam sputtering (IBS) by annealing the sample at $600^{\circ} \mathrm{C}$. Yang et al. [13] achieved a loss angle of $2.6 \times 10^{-4}$ in titania-tantala nanolaminates annealed at $650^{\circ} \mathrm{C}$, the titania nanolayer however becoming discontinuous.

In the present work, we investigate the effect of adding zirconia $\left(\mathrm{ZrO}_{2}\right)$ to the titanium-tantalum oxide. Zirconia, when alloyed with tantala, increases the maximum annealing temperature by frustrating the crystallization [14]. A higher annealing temperature is important as it allows for further material relaxation, thus leading to lower internal mechanical dissipation. Here, we show that codepositing several percent of zirconia and titania in tantala by magnetron sputtering produces dense 
and stoichiometric layers, that allows for a decrease in the loss angle down to $2.5 \times 10^{-4}$ upon annealing up to $800^{\circ} \mathrm{C}$, most importantly without inducing crystallization. However, the large difference in the coefficient of thermal expansion between the silica substrate and the thin film results in crack formation at high temperature that can be mitigated to some extent by capping the layers with silica.

\section{Experimental methodology}

\subsection{Magnetron sputtering}

Ternary alloys of zirconia-titania-tantala layers with a thickness of $850 \mathrm{~nm}$ on average (thickness ranging from 500 to $925 \mathrm{~nm}$ ) were co-deposited by reactive magnetron sputtering (MS) using a CMS-18 system from Kurt J. Lesker Co. Ltd. at École Polytechnique de Montréal. The substrates, 76-mm diameter, 1-mm-thick fused silica disks, were placed on a rotating stainless-steel substrate holder above three different metallic targets of $\mathrm{Zr}$, Ta and $\mathrm{Ti}$, at a distance of approximately $25 \mathrm{~cm}$. According to the supplier, the targets have a $99.95 \%$ purity. Prior to deposition, the pressure in the system was below

$1 \times 10^{-7}$ Torr. In order to pre-clean the samples, we applied a radio-frequency (RF) bias of $100 \mathrm{~V}$ to the substrate in a $\mathrm{O}_{2}$ :Ar gas flow of 1:2 at a pressure of 8 mTorr for 10 minutes. During deposition, the $\mathrm{O}_{2}$ :Ar was injected at a ratio of $6: 10$, using a total flow of $30 \mathrm{sccm}$ to maintain a pressure of 5 mTorr. The substrate holder was rotating to minimize thickness variations amounting to a radial non-uniformity of a few percent. Pieces of silica and crystalline silicon were placed next to the disk during deposition as witness samples.

Tantala films were prepared by reactive high power impulse magnetron sputtering (HiPIMS). Hala et al. [15] showed that this technique produces tantala layers with a higher refractive index than the more standard RF-MS. These layers also featured a lower residual stress than those deposited by RF-MS and ion beam sputtering techniques. For the two other components of the alloy, half of the $\mathrm{Ta}_{2} \mathrm{O}_{5}$ samples were doped with $\mathrm{ZrO}_{2}$ and $\mathrm{TiO}_{2}$ co-deposited using $\mathrm{RF}$ magnetron sputtering (RF-MS) on the Zr target and direct current magnetron sputtering (DC-MS) on the Ti target. For the other half, the power supplies powering the Ti and $\mathrm{Zr}$ targets were interchanged. The HiPIMS process was performed using a voltage of $700 \mathrm{~V}$ applied during $80 \mu \mathrm{s}$ at a frequency of $80 \mathrm{~Hz}$. The RF and DC voltages applied to the $\mathrm{Zr}$ and Ti targets varied between $200 \mathrm{~V}$ and $400 \mathrm{~V}$ in order to achieve different dopant concentrations. Depositions lasted 2 to 6 hours. 


\section{$2.2 \quad$ RBS and ellipsometry}

Rutherford backscattering spectrometry (RBS) was carried out on a 1.7 MV Tandetron accelerator at Université de Montréal using a He beam to determine the composition and areal atomic density of the thin films deposited on the Si witness samples. The measurements were done at two energies: 2 $\mathrm{MeV}$ so the spectrum analysis can be based on the Rutherford cross-section of oxygen, and $3.9 \mathrm{MeV}$ to better resolve the $\mathrm{Ta}, \mathrm{Zr}, \mathrm{Ti}$ and $\mathrm{Ar}$ contributions. The incidence angle was $7^{\circ}$ to decrease the chances of channeling in the Si substrate, and the scattering angle was $170^{\circ}$ to maximize the mass resolution. In order to determine the areal atomic density and the composition, simulations were carried out using SIMNRA [16]. The uncertainties were estimated by varying composition and areal density in the simulation until it no longer fitted the experimental data. For a given sample, the solution fitted the spectra acquired at both beam energies.

Spectrally-resolved ellipsometry was carried out on the fused silica disks, using a RC2-XI system from J.A. Woollam Co., Inc. at École Polytechnique de Montréal. Measurements covered wavelengths from 210 to $2500 \mathrm{~nm}$. The analysis was made by comparing the experimental $\Psi$ and $\Delta$ spectra to models generated using the CompleteEASE software to determine the film thickness and the complex refractive index as a function of wavelength. The optical properties were modeled using a general oscillator consisting of a Tauc-Lorentz and a Gaussian. The Gaussian was required in order to reproduce a more peaked, more intense UV absorption than allowed by a Tauc-Lorentz model. An optical model for the substrate was determined using a virgin fused silica substrate. A single set of optical parameters was determined for each deposited film using the data acquired at three locations and four incidence angles: $45^{\circ}, 55^{\circ}, 65^{\circ}$ and $75^{\circ}$. During the optimization, the parameters were bound to avoid nonphysical results, for example the amplitude of the oscillators was prevented from having a negative value. Then, in order to obtain the coating thickness distribution over the disk, measurements at 48 different positions using a mapping stage and at an incidence angle of $70^{\circ}$ were performed by only fitting the thickness and surface roughness, while keeping the optical model constant. The mapping revealed a $3 \%$ decrease of the deposited layer thickness between the center and the edges of the $75 \mathrm{~mm}$ silica substrates.

\subsection{Internal mechanical dissipation}

The internal mechanical dissipation was measured under high vacuum by electrostatically driving resonance modes on a gentle nodal suspension (GeNS) setup at California Institute of Technology [17]. 
Here, each coated silica disk rests in stable equilibrium centered on a curved Si surface. This minimizes sample clamping losses for any modes featuring a node at the disk center. The decay in amplitude of the resonance modes, between 1 and $30 \mathrm{kHz}$, is monitored as a function of time $t$ and is fitted with the following expression:

$$
A(t)=A_{0} e^{-\pi t f \phi_{\text {total }}},
$$

where $\phi_{\text {total }}$ is the measured loss angle for a given (coated) disk at frequency $f$. Prior to film deposition, the loss angle of the same silica disk, $\phi_{0}$, was measured, with typical values in the range of $10^{-7}$. Knowing the films' density (see below), and their thickness from ellipsometry mapping, a finite element method (FEM) calculation allows one to compute the dilution factor $D$ for each mode, which represents the ratio of elastic energy stored in the film compared to that of the substrate. We can then extract, $\phi_{c}$, the coating loss angle as follows:

$$
\phi_{c}=\left[\phi_{\text {total }}+(D-1) \phi_{0}\right] / D
$$

Since the film's thickness is typically less than $1 \mu \mathrm{m}$ while the substrate is $1 \mathrm{~mm}$ thick, $D \sim 10^{-3}$ and as $\phi_{c}$ is the subtraction of two similar numbers, divided by a small number, the resulting value bears a relatively large uncertainty.

It is usually found that the loss angle value has a mild dependency on frequency, which is a confirmation that the method provides a measurement of the internal mechanical dissipation, as other effects (e.g. edge-related dissipation) would be mode-dependent [18]. The loss angle values reported here are averages of all modes for frequencies ranging from $1 \mathrm{kHz}$ to $30 \mathrm{kHz}$. The error bars represent the standard deviation. Thanks to the FEM modeling, the Young's modulus and Poisson's ratio of the film can also be estimated from the resonance mode frequency shift between measurements carried out on virgin and coated substrates.

Following the loss-angle measurement on the as-deposited disks, each disk was annealed in air by steps of $50-100^{\circ} \mathrm{C}$ from $400^{\circ} \mathrm{C}$ to the point where it showed signs of crystallization. For each annealing step, the temperature was increased at $100^{\circ} \mathrm{C} /$ minute up to the annealing temperature, which was maintained for 10 hours, and then the samples cooled at $100^{\circ} /$ hour down to $40^{\circ}$. The loss angle was remeasured after each annealing step, also allowing one to observe changes in the Young's modulus. Crystallization manifests itself by a texture visible under the optical microscope and marked changes 


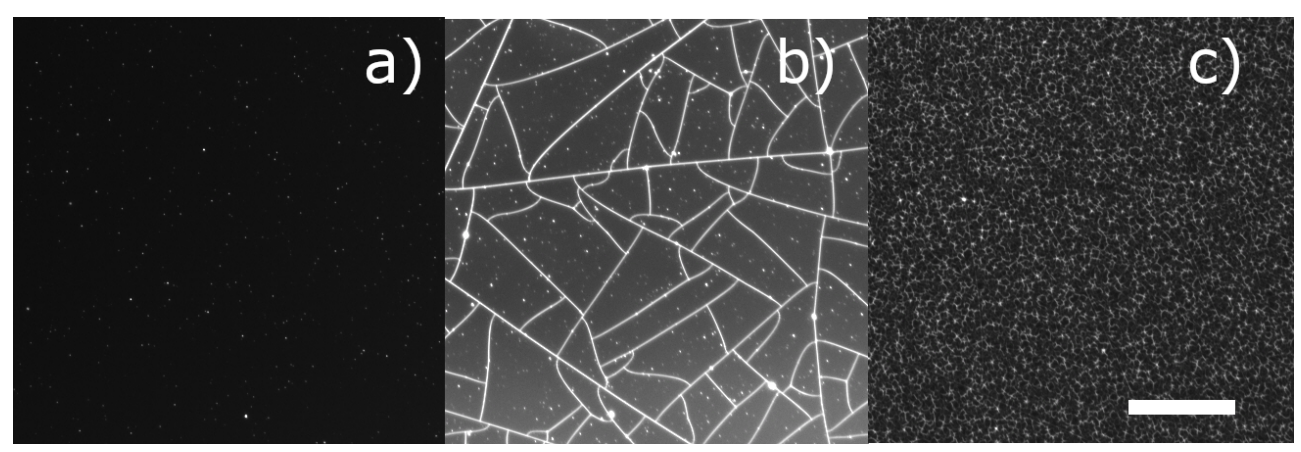

Figure 1: Micrographs of sample \#702 as-deposited (a) and after annealing at $700^{\circ} \mathrm{C}(\mathrm{b})$ and $750^{\circ} \mathrm{C}(\mathrm{c})$. The bar at bottom of panel c) is $50 \mu \mathrm{m}$ long.

in the mechanical properties and loss angle determined by the simulations. Figure 1 shows a series of dark-field optical micrographs at different stages of annealing for sample \#702, obtained using a Nikon Eclipse LV100 microscope. The as-deposited sample (a) appears uniform, although dark field microscopy highlights what we think are particles of dust. Cracking (b) will be discussed in the last section. The microstructure resulting from crystallization is clearly visible in panel c). In such samples, peaks related to the crystalline phase also showes up in Raman spectroscopy [19].

\section{Results and discussion}

\subsection{Mass density and stoichiometry}

The mass density is calculated by combining the RBS and ellipsometry measurements. Both measurements were carried out at the same location on the Si witness sample in order to avoid any influence of thickness gradient. Areal atomic density $\left(\right.$ at. $\left./ \mathrm{cm}^{2}\right)$ and composition found by RBS are used to determine the areal mass density $\left(\mathrm{g} / \mathrm{cm}^{2}\right)$. This result is then divided by the thickness obtained by ellipsometry, which gives us the mass density of the sample $\left(\mathrm{g} / \mathrm{cm}^{3}\right)$. The uncertainty on the number of at $/ \mathrm{cm}^{2}$ measured by RBS was typically under $3 \%$ while the one on thickness deduced from ellipsometry was typically $0.5 \%$.

Figure 2a) presents the density of the samples as a function of the $\mathrm{Ti}$ and $\mathrm{Zr}$ cation fraction in the alloy. The blue plane shows the rule of mixture, i.e., a linear interpolation between the densities of pure $\mathrm{Ta}_{2} \mathrm{O}_{5}[20]$, pure $\mathrm{ZrO}_{2}[21]$ and pure $\mathrm{TiO}_{2}[22,23]$ according to their respective proportion in the alloy. Figure $2 \mathrm{~b}$ ) is the same figure tilted parallel to the blue plane. We see, within the uncertainties, that most films follow the calculated bulk density.

The oxygen content, determined by RBS measurements with a $2 \mathrm{MeV}$ He beam, is plotted as a 

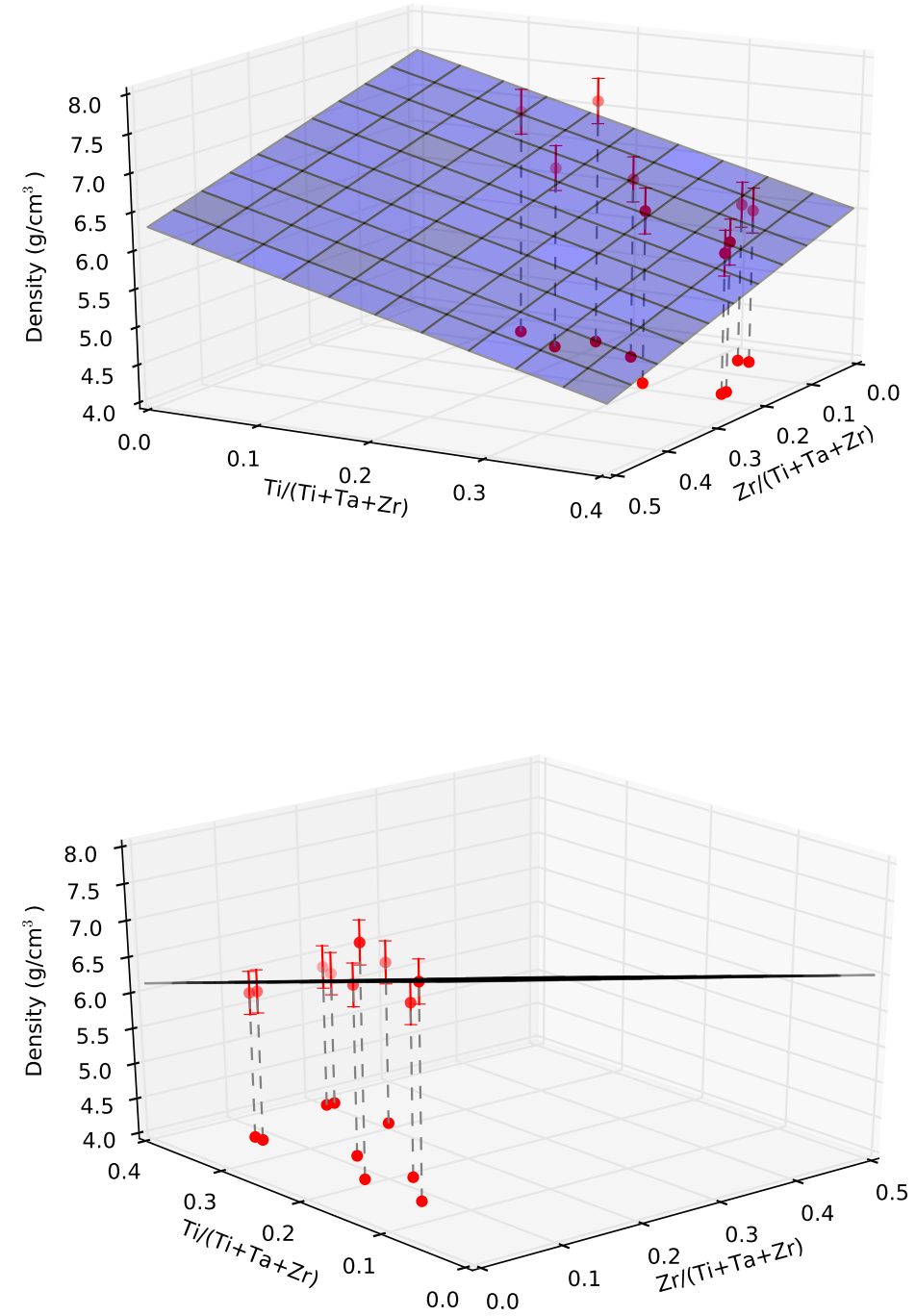

Figure 2: a) Mass density as a function of $\mathrm{Zr}$ and $\mathrm{Ti}$ contents. The blue plane is the expected density assuming a linear interpolation between the bulk densities of pure $\mathrm{Ta}_{2} \mathrm{O}_{5}$ [20] $\left(7.7 \mathrm{~g} / \mathrm{cm}^{3}\right)$, pure $\mathrm{ZrO}_{2}[21]\left(5.3 \mathrm{~g} / \mathrm{cm}^{3}\right)$ and pure $\mathrm{TiO}_{2}[22,23]\left(3.8 \mathrm{~g} / \mathrm{cm}^{3}\right)$. b) Same figure but oriented parallel to the plane. 
function of doping in Fig. 3. Within uncertainties, it follows the expected interpolation between the fraction of oxygen in $\mathrm{Ta}_{2} \mathrm{O}_{5}(\sim 71.4 \%)$ and $(\mathrm{Ti}, \mathrm{Zr}) \mathrm{O}_{2}(\sim 66.7 \%)$, shown as a black line in the figure.

These results confirm that sputter-deposition involving HiPIMS produces stoichiometric films with bulk-like density, hence good quality layers, which should also feature fewer defects. The above observation results from the fact that the HiPIMS process generates a highly ionized plasma leading to a high kinetic energy and high flux of species arriving at the substrate, giving rise to compact films with lower stress [15]. In addition, residual stress in the films can further be relieved upon annealing. In turn, we expect HiPIMS-deposited films to feature optical properties tending towards bulk-like values.

Indeed, in Table 1 we report the refractive index at $550 \mathrm{~nm}$ and $1064 \mathrm{~nm}$ (the wavelength of the laser in a-LIGO), retrieved from the optical model found for each sample during the ellipsometry spectra analysis. They are compared with the value expected assuming the rule of mixture computed using the refractive index found in Refs. [15], [24], and [25] for HiPIMS-deposited $\mathrm{Ta}_{2} \mathrm{O}_{5}$, bulk yttria-stabilized $\mathrm{ZrO}_{2}$, and $\mathrm{TiO}_{2}$ deposited by IBS, respectively. We see that our refractive index values match those deduced from the rule of mixture, although they are generally slightly lower, by $1.5 \%$ on average.

It is worth pointing out that, especially for titania, a wide range of values are reported in the literature for its refractive index, depending on the deposition method and post-treatment [25]. KlembergSapieha et al. [6], carrying out deposition of tantala, titania and silica by various methods, point out that titania microstructural evolution and nucleation process is especially sensitive to the energetic conditions, resulting in films featuring anatase or rutile crystalline phases and their corresponding higher refractive index under high ion flux conditions. By interpolation using the values obtained by Magnozzi et al. for titania [25], we conclude that the results are comparable to a material that is still amorphous while being obtained by an energetic deposition method such as IBS that typically produces dense, high-quality films.

\subsection{Loss angle}

Figure 4 a) shows the evolution of the loss angle as a function of the annealing temperature for four representative samples of our set: low $\mathrm{Ti}$ and $\mathrm{Zr}$ doping (yellow squares), intermediate $\mathrm{Ti}$ and $\mathrm{Zr}$ doping (orange diamonds), high $\mathrm{Ti}$ and $\mathrm{Zr}$ doping (blue circles), and high Ti doping with low $\mathrm{Zr}$ doping (green triangles). For comparison, the dashed line indicates the loss angle of the high-index layers in the current a-LIGO Bragg reflectors $\left(25 \% \mathrm{TiO}_{2}, 75 \% \mathrm{Ta}_{2} \mathrm{O}_{5}\right)[26]$. The red symbols indicate films that have likely crystallized as their loss angle value and Young's modulus, deduced from the frequency shift of the modes, show a significant departure from the general trend, in addition to the 


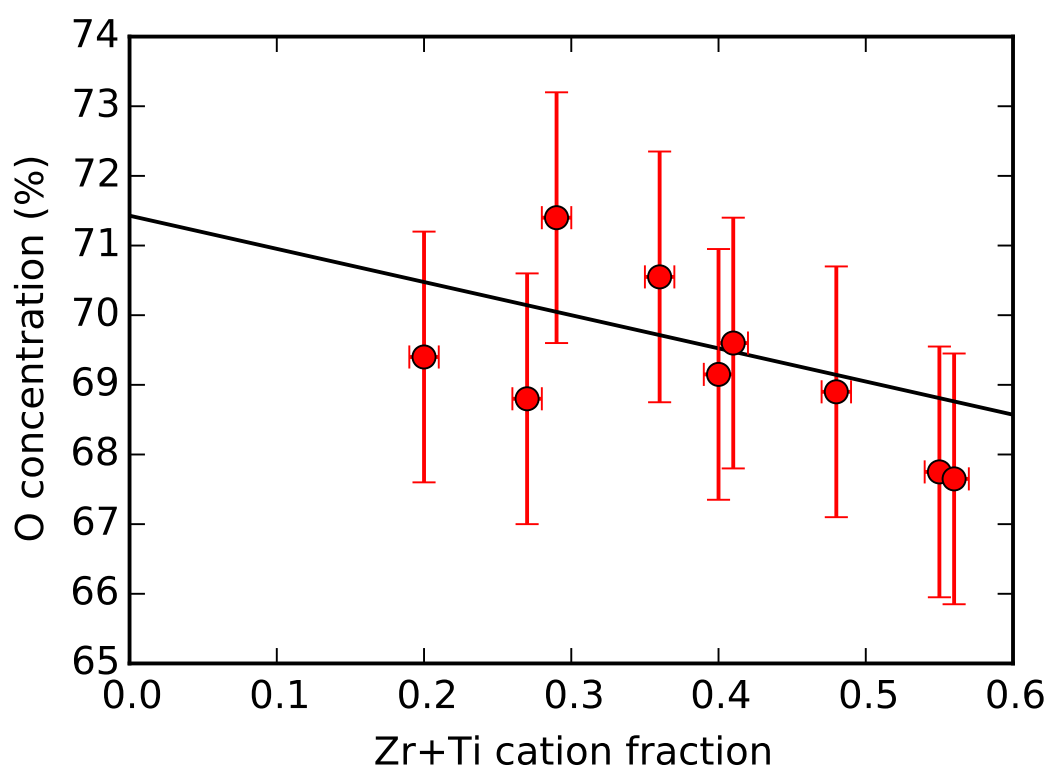

Figure 3: Atomic concentration of oxygen, found by RBS, as a function of dopant cation fraction. The black line is the expected value relating $\mathrm{Ta}_{2} \mathrm{O}_{5}$ $(5 / 7)$ and $\mathrm{ZrO}_{2}+\mathrm{TiO}_{2}(2 / 3)$.

films presenting a surface texture under optical microscopy (such as in Fig. 1c). This is the case of the films with the lowest doping (which crystallized between $700^{\circ} \mathrm{C}$ and $750^{\circ} \mathrm{C}$ ), and the intermediate doping (between $750^{\circ} \mathrm{C}$ and $800^{\circ} \mathrm{C}$ ).

It is seen that the different as-deposited samples feature a similar loss angle within uncertainty. The same also applies after an anneal at $400^{\circ} \mathrm{C}$, however, above this temperature, a departure between the different data sets is observed. While not showing convincing signs of crystallization, the sample with high $\mathrm{Ti}$ and low $\mathrm{Zr}$ doping (green triangles) features an increase of its loss angle above $700^{\circ} \mathrm{C}$. The sample that has the highest $\mathrm{Zr}+\mathrm{Ti}$ doping shows the lowest loss angle, $2.5 \times 10^{-4}$, although only marginally lower than the sample with intermediate doping annealed at $750^{\circ} \mathrm{C}$, before it crystallizes (yellow diamonds). Still the data shows a clear decrease in the loss angle both with increasing annealing temperature and increasing doping.

In order to assess the effect of doping at a given temperature, the value of the loss angle is plotted as a function of the $\mathrm{Zr}$ and $\mathrm{Ti}$ content in Fig. $4 \mathrm{~b}$ ), after annealing the samples at $650^{\circ} \mathrm{C}$, a temperature at which none of the ternary samples has shown signs of crystallization. The figure includes values from zirconia-doped tantala, binary sample from a separate study (green triangles) [19]. The blue 

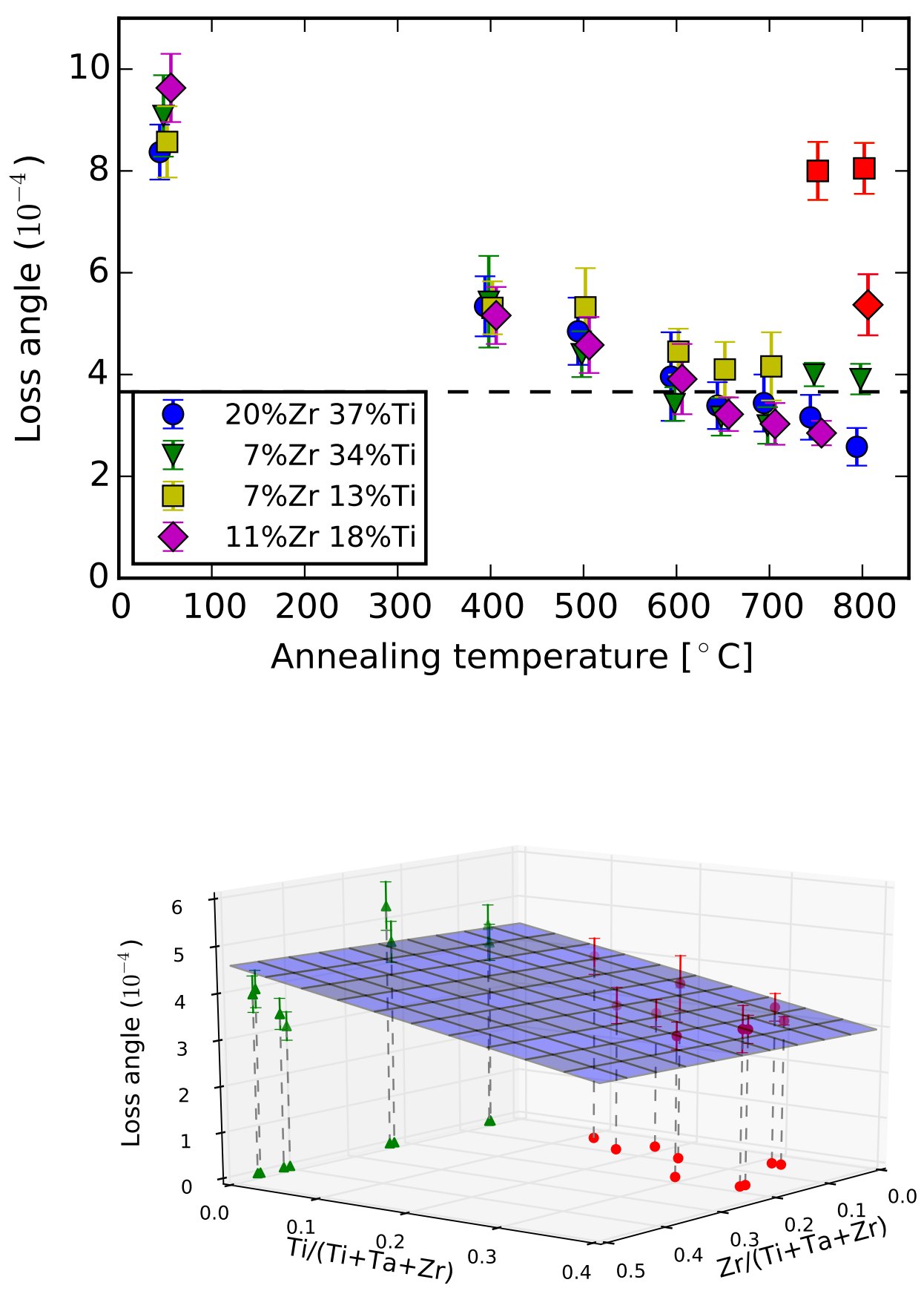

Figure 4: a) Loss angle as a function of annealing temperature for a subset of the deposited samples, representative of the different levels of doping achieved, as listed in the legend. Red symbols indicate samples that have likely crystallized. Data slightly shifted horizontally for clarity. b) Loss angle after annealing at $650^{\circ} \mathrm{C}$ as a function of doping concentration. The green triangles are binary (without $\mathrm{Ti}$ ) sample for previous studies [19] and the red circles are the ternary sample. The blue plane is a bi-linear regression of the data. 


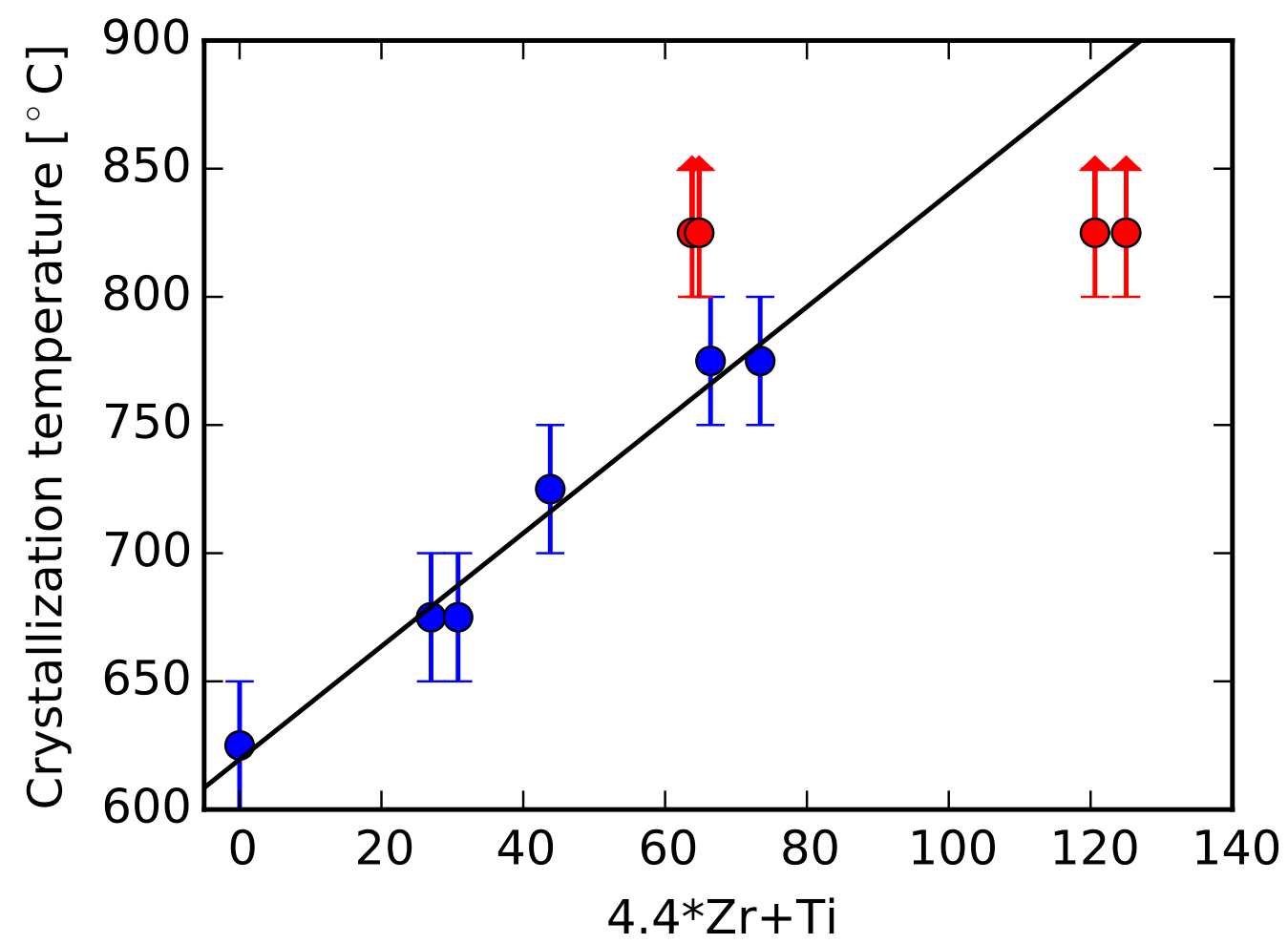

Figure 5: Crystallization temperature, $T_{C}$, as a function of $\mathrm{Zr}$ and $\mathrm{Ti}$ concentration. To broaden the values, non-doped tantala samples, and Ti- only or Zr- only doped tantala samples were also considered to determine the contribution of $\mathrm{Zr}$ and $\mathrm{Ti}$ to the crystallization temperature. The weight on the $\mathrm{Zr}$ concentration (4.4) is also fitted in order to minimize the squared deviation to the fit (black line). The red points are the samples which did not show signs of crystallization at $800^{\circ} \mathrm{C}$, and are not included in the fit. 
plane is a bi-linear regression represented by the following equation :

$$
\phi_{c}=[(4.2 \pm 0.7)+(1 \pm 2) x-(4 \pm 2) y] \times 10^{-4}
$$

where $x$ and $y$ are the cation fractions of $\mathrm{Zr}$ and $\mathrm{Ti}$, respectively. The uncertainties are the standard error on the coefficients. Loss angles of zirconia-doped tantala samples (no Ti) from a separate study [19] were also considered for this analysis. According to the parameters of the plane equation, $\mathrm{Ti}$ reduces the loss angle, as previously observed [8], while the $\mathrm{Zr}$ concentration does not, since the $x$ coefficient is compatible with 0 , given the uncertainty. While $\mathrm{Zr}$ concentration does not directly reduce the loss angle at a given temperature, it still allows one to reach a higher annealing temperature without signs of crystallization, as we've seen in Fig. 4 a) for samples containing more Zr.

In Table 1, the crystallization temperature, $T_{C}$, is shown as a function of $\mathrm{Zr}$ and Ti content. We see that the combination of $\mathrm{Zr}$ and $\mathrm{Ti}$ increases $T_{C}$. While $28 \%$ of $\mathrm{Ti}$ increases that temperature to $675^{\circ} \mathrm{C}$, a few additional percent of $\mathrm{Zr}$ allows it to reach as much as $800^{\circ} \mathrm{C}$.

In order to clarify the respective role of $\mathrm{Zr}$ and $\mathrm{Ti}$ in increasing $T_{C}$, a linear minimization of the crystallization temperature as a function of the dopant concentration is presented in Fig. 5. A better correlation between $T_{C}$ and the dopant content is found if a larger weight is given to the $\mathrm{Zr}$ content. A $\chi^{2}$ minimization that includes a multiplying factor for the $\mathrm{Zr}$ fraction indicates that it affects $T_{C}$ 4.4 times more than Ti. Indeed, the reason for incorporating $\mathrm{Zr}$ was to frustrate crystallization [14], and hence to allow annealing at higher temperatures. Our results show that, indeed, zirconia doping is much more effective than titania doping with regards to the frustration of tantala crystallization.

Added as red symbols to Fig. 5 are the samples that did not show signs of crystallization even at $800^{\circ} \mathrm{C}$, and thus were ignored from the fit; in agreement with what we discussed, these are the samples with the highest dopant concentration. Hence, more doping allows, in general, for higher annealing temperatures, which in turn helps decrease the loss angle.

However, the improvement obtained by annealing at temperatures above $750^{\circ}$ in terms of loss angle appears to be marginal in comparison to the loss angle of current a-LIGO mirrors and recent results from the literature for titania-doped tantala $[12,13]$, and involves annealing the eventual mirrors at extremely high temperatures, $>800^{\circ} \mathrm{C}$, with potential problems such as cracking. 


\begin{tabular}{|c|c|c|c|c|c|c|c|}
\hline \multirow{3}{*}{ Sample } & \multirow{3}{*}{$\mathrm{Zr}[\%]$} & \multirow{3}{*}{$\mathrm{Ti}[\%]$} & \multirow{3}{*}{ Cryst. temp. $\left[ \pm 25^{\circ} \mathrm{C}\right]$} & \multicolumn{4}{|c|}{ Index of refraction } \\
\hline & & & & \multicolumn{2}{|c|}{$550 \mathrm{~nm}$} & \multicolumn{2}{|c|}{$1064 \mathrm{~nm}$} \\
\hline & & & & Ellipso. & Rule Mix. & Ellipso. & Rule Mix. \\
\hline 657 & 0 & 0 & 625 & 2.18 & 2.20 & 2.12 & 2.13 \\
\hline 660 & 0 & 28 & 675 & 2.23 & 2.26 & 2.15 & 2.18 \\
\hline $546[19]$ & 7 & 0 & 675 & 2.17 & 2.20 & 2.11 & 2.13 \\
\hline 662 & 20 & 30 & - & 2.25 & 2.28 & 2.27 & 2.19 \\
\hline 697 & 19 & 37 & $>800$ & 2.25 & 2.28 & 2.17 & 2.20 \\
\hline 698 & 20 & 37 & $>800$ & 2.25 & 2.28 & 2.18 & 2.20 \\
\hline 699 & 7 & 33 & $>800$ & 2.21 & 2.28 & 2.14 & 2.19 \\
\hline 700 & 7 & 34 & $>800$ & 2.20 & 2.28 & 2.13 & 2.19 \\
\hline 701 & 7 & 20 & - & 2.17 & 2.25 & 2.11 & 2.17 \\
\hline 702 & 7 & 13 & 725 & 2.18 & 2.23 & 2.12 & 2.15 \\
\hline 703 & 11 & 18 & 775 & 2.18 & 2.24 & 2.12 & 2.17 \\
\hline 704 & 11 & 25 & 775 & 2.18 & 2.26 & 2.11 & 2.18 \\
\hline
\end{tabular}

Table 1: Crystallization temperature and index of refraction for different cation fractions of zirconium and titanium. Some samples ("-") were not annealed and were kept for future reference. Refractive indices, obtained prior to annealing, are compared to the value obtained assuming the rule of mixture and considering the refractive index from Ref. [15] for HiPIMS-deposited tantala (2.20 and 2.13 at 550 and $1064 \mathrm{~nm}$, respectively), Ref. [24] for bulk yttria-stabilized zirconia (2.17 and 2.12), and Ref.[25] for titania deposited by IBS (2.44 and 2.32). The first three lines are data for non-ternary samples for references.

\subsection{Cracking}

Indeed, a side effect of the annealing at very high temperature is that it leads to crack formation, as observed in Fig. 1b). High-index materials usually feature a coefficient of thermal expansion (CTE) which is much larger than that of the silica substrate, and may crack as a result of the amount of stress induced by the difference of CTE. Here, the CTE is $2.45 \times 10^{-6} \mathrm{~K}^{-1}$ and $2.57 \times 10^{-6} \mathrm{~K}^{-1}$ for tantala and titania, respectively [27], a difference of more than $2 \times 10^{-6} \mathrm{~K}^{-1}$ compared to silica at $0.38 \times 10^{-6} \mathrm{~K}^{-1}[27]$. It is worth pointing out that, while in a 1064-nm Bragg reflector high-index layers are $\sim 130 \mathrm{~nm}$ thick, for the purpose of minimizing the loss angle measurement uncertainty, thicker films $(\sim 850 \mathrm{~nm})$ were deposited in our experiments, aggravating the issue. As a result, most films deposited in this study cracked upon annealing before reaching the crystallization temperature.

It is interesting to note that cracks do not appear to significantly affect the loss angle measurement, as no abrupt change is visible in the loss angle data. In fact, it was shown that interfaces, namely those between the deposited film and the substrate, are not important contributors to the loss angle in our geometry [18]. In this sense, cracks only add a few percent to the amount of interface compared 
to the amount of layer-substrate interface. For example, for a $1 \mu \mathrm{m}$ film, a square network of cracks $50 \mu \mathrm{m}$ apart covering the whole surface of the sample would only represent $\sim 4 \%$ of the film-substrate interface. It is also true that the nature of this interface might be quite different. This issue is discussed further below.

In a mirror stack, the high-index material is alternated with silica layers, the latter being thicker. Such a configuration should constrain an expanding high-index layer from both the top and the bottom, and thus may help avoid cracking. In order to see if the maximum annealing temperature before cracking is increased in such a configuration, we deposited a silica film of $506 \mathrm{~nm}$ by RF-MS on top of a $416 \mathrm{~nm}$ ternary film. As expected, the loss angle measured for this sample falls between that of silica and the ternary alloy. In order to deconvolute the respective loss angles, we additionally produced two reference samples with the same deposition parameters but on different disks, with twice the deposition time in order to obtain 1- $\mu$ m-thick layers. In the ternary alloy, the Ta:Ti: $\mathrm{Zr}$ cation proportion is 21:41:38, hence a relatively high doping. Unlike the results reported above for which the samples were synthesized by HiPIMS, the ternary layer was synthesized without HiPIMS to see the influence of that technique on the loss angle. The Ta and $\mathrm{Zr}$ targets were powered from the RF power supplies while the Ti target was powered from the DC power supply.

The latter samples were annealed by steps of $50^{\circ} \mathrm{C}$ from $500^{\circ} \mathrm{C}$ to $650^{\circ} \mathrm{C}$. Figure 6 is a representation of the loss angle as a function of the annealing temperature for these three samples. The ternary layer cracked between 500 and $550^{\circ} \mathrm{C}$ while the sample with the silica cap cracked approximately $100^{\circ} \mathrm{C}$ higher, as indicated by the two vertical lines. It is worth pointing out that the increase in the critical temperature of cracking could be a combined effect of the silica capping layer and the fact that the ternary layer in the bilayer is half the thickness than that of the single ternary layer. Hence, half the mechanical energy is stored in the bilayer.

Still, in this $100^{\circ} \mathrm{C}$ interval, the loss angle decreases by about $0.3 \times 10^{-4}$. The loss angle obtained at $650^{\circ} \mathrm{C}$ for this highly doped sample is slightly higher than what was obtained when the Ta was deposited by HiPIMS (blue circles in Fig. 4). Given the uncertainties, we cannot confirm that HiPIMS helped synthesize layers with less internal mechanical dissipation than RF-MS, despite the fact that it produced dense and stoichiometric films. After annealing, the silica layer reaches a loss angle of about $0.5 \times 10^{-4}$, compatible with what is usually observed for deposited silica.

As previously mentioned, the obtained loss angle of the bilayer (red squares in Fig. 6) is an intermediate value between the contributions of both the silica layer and the ternary layer. To properly 


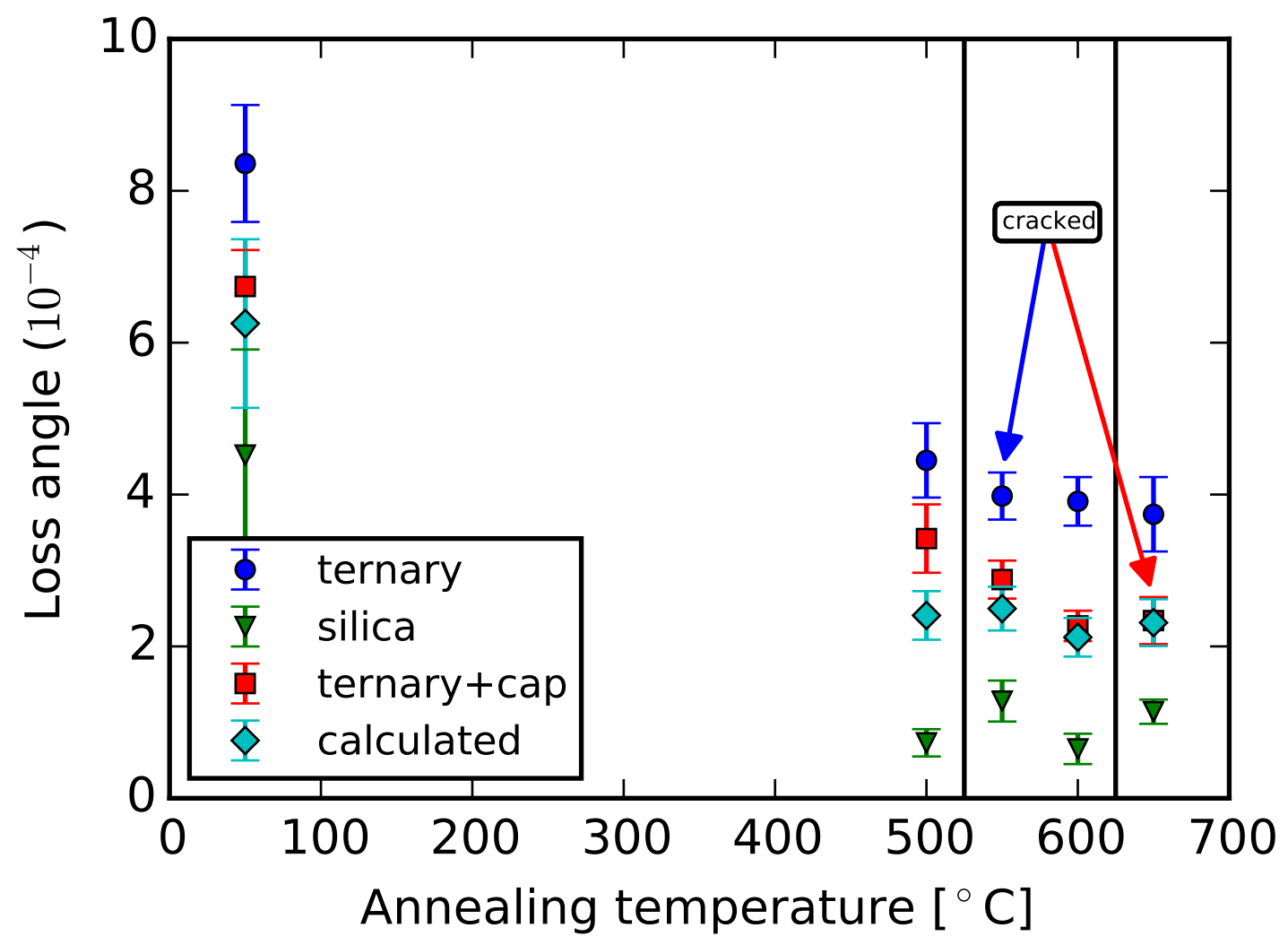

Figure 6: Loss angle as a function of annealing temperature. The red squares are the bilayer sample. The green triangles and blue circles are, respectively, the ternary and the silica monolayer samples. The cyan diamonds are the weighted average of the two monolayers weighted by their thickness in the bilayer. The perpendicular black lines represent the temperature of cracking at $525^{\circ} \mathrm{C}$ for the ternary monolayer and at $625^{\circ} \mathrm{C}$ for the bilayer.

analyze these results, we computed an average of the silica and ternary loss angles weighted by their relative thickness in the bilayer and we plot it as cyan diamonds in the Fig. 6 . We see that the loss angle of the capped sample and calculated data overlap within uncertainty. This also confirms that the presence of cracks does not influence significantly the loss angle and that we can consider the loss angle results of the cracked samples as reliable.

\section{Conclusion}

Titania-doped tantala is the material that was previously found to be the best compromise in terms high refractive index and of low loss angle $\left(3.66 \times 10^{-4}\right)$ while satisfying other requirements for the large optical components of gravitational wave observatories such as a-LIGO, especially for the mirrors. Internal mechanical losses are still one of the main sources of noise in the most sensitive frequency 
range of these experiments. Here, we show that incorporating zirconia to this binary alloy allows one to increase the annealing temperature before the onset of the materials' crystallization, from $650^{\circ} \mathrm{C}$ to $>800^{\circ} \mathrm{C}$, which in turn helps decrease the loss angle, down to $2.5 \times 10^{-4}$, an improvement by a factor 1.5 over current a-LIGO mirrors. Since the noise decreases as the square root of the loss angle, such an improvement would allow one to reduce this component of the noise by $\sim 20 \%$. We also show that zirconia doping is much more effective than titania doping in frustrating crystallisation of tantala.

The HiPIMS deposited $\mathrm{Ta}_{2} \mathrm{O}_{5}$ films feature densities and a stoichiometry equivalent to bulk values but the improvement in terms of lower internal mechanical dissipation is not conclusive in comparison to a RF-MS deposited film. Also, as a result of the higher annealing temperatures and the large difference in CTE between the ternary alloy and the silica substrate, most of the $\sim 850$ nm-thick samples cracked at around $500-550^{\circ} \mathrm{C}$. Producing a bilayer, comparable to the actual Bragg mirror stack in a-LIGO, allows one to increase by $100^{\circ} \mathrm{C}$ the temperature reached before the layers crack. Such an alloy is therefore a viable solution for this optics, although the gain in terms of reduced noise remains somewhat marginal.

\section{Acknowledgments}

The work performed at U. Montréal and Polytechnique Montréal was supported by the Natural Sciences and Engineering Research Council of Canada (NSERC), the Canadian foundation for innovation (CFI) and the Fonds de recherche Québec, Nature et technologies (FQRNT) through the Regroupement Québécois sur les matériaux de pointe (RQMP). The authors thank S. Roorda, M. Chicoine, R. Shink, and L. Godbout from U. Montréal and F. Turcot from Polytechnique Montréal for fruitful discussions and technical support. They also thank their colleagues within the LIGO Scientific Collaboration for advice and support. LIGO was constructed by the California Institute of Technology and Massachusetts Institute of Technology with funding from the National Science Foundation, and operates under cooperative agreement PHY-0757058. Advanced LIGO was built under award PHY0823459. M. Fejer and R. Bassiri acknowledge the support of the LSC Center for Coatings Research, jointly funded by the National Science Foundation (NSF) and the Gordon and Betty Moore Foundation (GBMF), in particular through NSF, PHY-1708175 and GBMF Grant No. 6793. This paper has LIGO document number LIGO-P2000523. The data that support the findings of this study are available from the corresponding author upon reasonable request. 


\section{References}

[1] B. P. Abbott et al., Phys. Rev. Lett. 116, 061102 (2016).

[2] B. P. Abbott et al., Phys. Rev. Lett. 119, 161101 (2017).

[3] B. P. Abbott et al., Phys. Rev. Lett. 116, 131103 (2016).

[4] Y. Levin, Phys. Rev. D 57, 659 (1998).

[5] N. Kaiser and H. K. Pulker, Optical Interference Coatings, Springer-Verlag Berlin Heidelberg, 2003.

[6] J. E. Klemberg-Sapieha et al., Appl. Opt. 43, 2670 (2004).

[7] E. Çetinörgü, B. Baloukas, O. Zabeida, J. E. Klemberg-Sapieha, and L. Martinu, Appl. Opt. 48, 4536 (2009).

[8] G. M. Harry et al., Classical and Quantum Gravity 24, 405 (2006).

[9] M. Granata et al., Classical and Quantum Gravity 37, 095004 (2020).

[10] S. Reid and I. Martin, Coatings 6, 61 (2016).

[11] G. Vajente et al., Classical and Quantum Gravity 35, 075001 (2018).

[12] M. A. Fazio et al., Opt. Mater. Express 10, 1687 (2020).

[13] L. Yang et al., ACS Applied Nano Materials 3, 12308 (2020).

[14] J.-Y. Tewg, Y. Kuo, and J. Lu, Electrochemical and Solid-State Letters 8, G27 (2005).

[15] M. Hála, R. Vernhes, O. Zabeida, J.-E. Klemberg-Sapieha, and L. Martinu, Journal of Applied Physics 116, 213302 (2014).

[16] M. Mayer, SIMNRA User's Guide, 1997.

[17] G. Vajente et al., Review of Scientific Instruments 88, 073901 (2017).

[18] S. D. Penn et al., Physics Letters A 352, 3 (2006).

[19] M. Abernathy et al., Exploration of zirconia-doped-tantala films for gravitational wave detectors, submitted, 2020. 
[20] R. Bassiri et al., Applied Physics Letters 98, 031904 (2011).

[21] D. Ceresoli and D. Vanderbilt, Phys. Rev. B 74, 125108 (2006).

[22] B. Prasai, B. Cai, M. K. Underwood, J. P. Lewis, and D. A. Drabold, Journal of Materials Science 47, $7515(2012)$.

[23] D. Lide, CRC Handbook of Chemistry and Physics 77th edition ed DR Lide, Boca Raton: CRC Press, 1997.

[24] D. L. Wood and K. Nassau, Appl. Opt. 21, 2978 (1982).

[25] M. Magnozzi et al., Optical Materials 75, 94 (2018).

[26] M. Principe et al., Phys. Rev. D 91, 022005 (2015).

[27] C.-C. Lee, C.-L. Tien, W.-S. Sheu, and C.-C. Jaing, Review of Scientific Instruments 72, 2128 (2001). 